\title{
Anterolateral Meniscofemoral Ligament of the Lateral Meniscus
}

\author{
Young-Mo Kim, $\mathrm{MD}^{1}$, Yong-Bum Joo, $\mathrm{MD}^{1}$, Kyu-Woong Yeon, $\mathrm{MD}^{2}$, and Ki-Young Lee, $\mathrm{MD}^{1}$ \\ ${ }^{1}$ Department of Orthopedic Surgery, Chungnam National University School of Medicine, Daejeon; ${ }^{2}$ Department of Orthopedic Surgery, Daejeon Hankook Hospital, \\ Daejon, Korea
}

\begin{abstract}
Anatomical variations of the meniscus are a common anomaly that knee surgeons frequently encounter. However, anomalies of the anterior horn of the lateral meniscus (AHLM) are extremely rare. In this report, we present a newly discovered anomaly of the AHML: an anterolateral meniscofemoral ligament is described with clinical features and radiographic and arthroscopic findings.
\end{abstract}

Keywords: Knee, Lateral meniscus, Meniscofemoral ligament

The normal meniscus is completely attached to the tibia anteriorly and posteriorly, which generates hoop stress during axial loading and provides shock absorption ${ }^{1,2)}$. The meniscofemoral ligament (MFL), a small fibrous ligament of the knee joint, is a well-known anomaly that arises from the posterior horn of the lateral meniscus (PHLM) and attaches to the lateral area of the medial condyle of the femur. The anterior MFL is known as the ligament of Humphrey that crosses superiorly and medially behind the posterior cruciate ligament (PCL), and the posterior MFL is known as the ligament of Wrisberg that passes superiorly and medially in front of the PCL ${ }^{3-5)}$.

Unlike the anomalies of the PHLM, anomalies of the anterior horn of the lateral meniscus (AHLM) have been very rarely described: only 1 case has been reported where the subject lacked tibial attachment of the AHLM, which was also accompanied by anterior cruciate ligament (ACL) deficiency ${ }^{6}$.

Received June 17, 2015; Revised August 10, 2015;

Accepted August 11, 2015

Correspondence to: Yong-Bum Joo, MD

Department of Orthopedic Surgery, Chungnam National University

School of Medicine, 266 Munhwa-ro, Jung-gu, Daejeon 35015, Korea

Tel: +82-42-338-2412, Fax: +82-42-252-7098

E-mail: longman76@hanmail.net

This is an Open Access article distributed under the terms of the Creative Commons Attribution Non-Commercial License (http://creativecommons.org/licenses/by-nc/4.0/) which permits unrestricted non-commercial use, distribution, and reproduction in any medium, provided the original work is properly cited.
In this report, we describe a previously unreported case of an anterolateral MFL as an anomaly of the AHLM with an intact ACL: the anterolateral and anteromedial MFLs were merged into a single ligamentous structure. We also present detailed magnetic resonance image (MRI) and arthroscopic findings as well as discuss this condition in the context of previous reports.

\section{Case Report}

An 18-year-old male visited our hospital complaining of right knee pain that had lasted for 7 months. The patient was $182.1-\mathrm{cm}$ tall and weighed $103.4 \mathrm{~kg}(228 \mathrm{lb})$ and was a wrestler who had been active in athletics for over 10 years. On physical examination, the joint was negative for locking, snapping, and giving way and showed a normal range of motion; however, he had been experiencing a catching sensation for 1 year. Dull pain was localized on the anterolateral side of the right knee, and there was tenderness in the same region. His visual analog scale score was usually 2 , but was $\geq 6$ when aggravated. Preoperative MRI showed an approximately 5-mm radial tear of the PHLM (Fig. 1). Given the patient's physique, athletic activity (wrestling), and the size of the PHLM tear, we thought that the radial tear of the PHLM would become further aggravated and may cause additional injuries to surrounding cartilages, and decided to perform an arthroscopic examination.

Arthroscopy showed two ligament structures that arose from the anterior horn of the medial meniscus (AHMM) and AHLM, 
respectively and were attached to the intercondylar notch of the lateral condyle of the femur. These two ligaments were combined together, extended superiorly, ran parallel to the ACL, and then inserted into the intercondylar notch in the 12 oclock direction (Fig. 2A). Describing this combined ligament separately, the anteromedial MFL, which started from the AHMM, appeared to have the typical pattern that surgeons can occasionally observe ${ }^{4)}$ (Fig. 2B), and the anterolateral MFL, which had never been found or reported, started from the AHLM, ran parallel to the $\mathrm{ACL}$, and inserted into the superior portion of the intercondylar notch anterior to the ACL (Fig. 2C). Because the AHLM was not

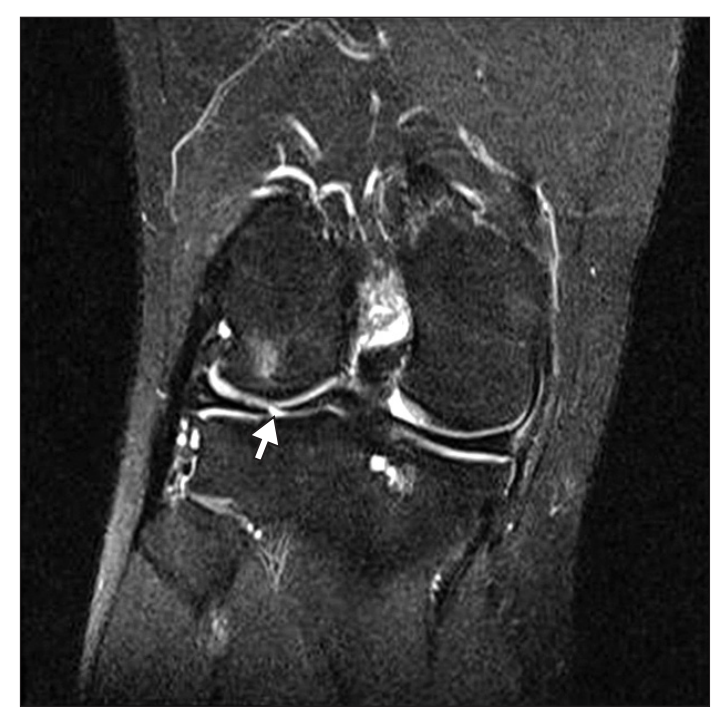

Fig. 1. Coronal T2-weighted magnetic resonance imaging scan showing a radial tear of the posterior horn of the lateral meniscus (white arrow). attached to the tibia, hypermobility occurred and led to degeneration of the AHLM. In addition, an Outerbridge grade II cartilage injury was visible on the anterior portion of the tibial plateau (Fig. 3). On the postoperative MRI, a fibrous structure assumed to be the anterolateral MFL and the anteromedial MFL were identified (Fig. 4). Although the characteristics of the patient's pain were inconsistent with PHLM radial tears, such an injury can completely disable the load-bearing function of the meniscus and thus result in an articular cartilage injury, wear, and degeneration. Thus, an arthroscopic partial meniscectomy for the radial tear of the PHLM was performed. At 1-year short-term outpatient followup, the patient complained of sustained anterolateral joint line pain.

\section{Discussion}

Appropriate anatomic placement of the tibial meniscus attachment is necessary to preserve critical knee joint functions ${ }^{7}$. Therefore, a deformed tibial meniscus attachment would disable these functions.

The anteromedial MFL that is an anomaly of the AHMM has been descried by some authors ${ }^{8)}$ and they have contended that AHMM hypermobility can lead to wear and subsequent degenerative changes ${ }^{9}$. However, there has been only 1 reported case of anterolateral MFL that was found in a patient with congenital absence of the ACL, indicating how extremely rare the anomaly is ${ }^{6}$.

On review of the present case's MRI, ligamentous structures were identified anterolateral to the normal ACL, which demonstrated low signal intensity on sagittal T1- and T2-weighted MRI
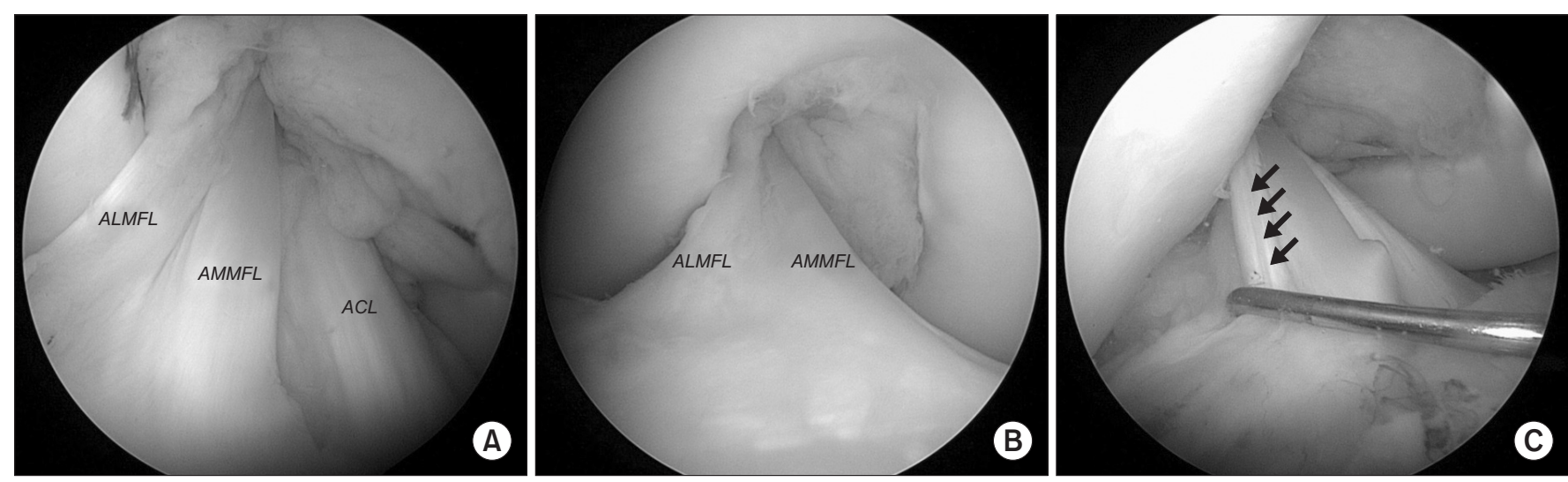

Fig. 2. (A) Arthroscopic view through the mid-patellar portal. The anteromedial meniscofemoral ligament (AMMFL) and anterolateral meniscofemoral ligament (ALMFL) merge into a single structure while running toward the intercondylar notch. (B) Arthroscopic view through the anterolateral portal. The AMMFL and ALMFL arise from the anterior horn of the medial and lateral meniscus, insert into the intercondylar notch anterior to the anterior cruciate ligament (ACL). (C) Arthroscopic view of the anterolateral meniscofemoral ligament (black arrows) through the anterolateral portal. It arises from the anterior horn of the lateral meniscus and extends along with the ACL before finally inserting at the posteromedial portion of the intercondylar notch. 

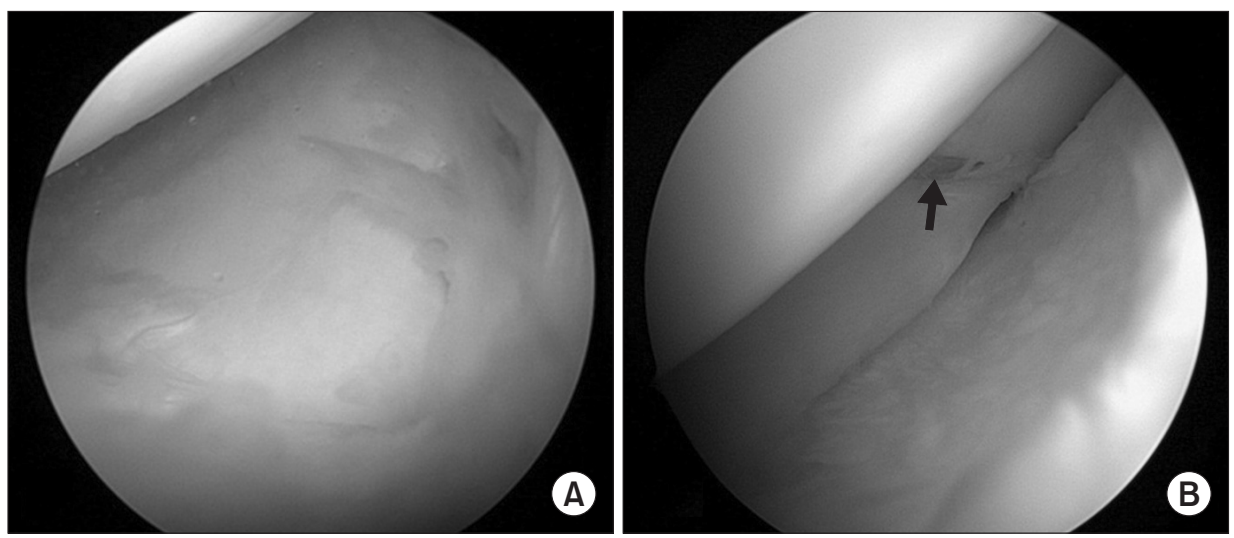

Fig. 3. Arthroscopic views through the anterolateral portal showing lateral meniscus degeneration (A) and a radial tear of the posterior horn of the lateral meniscu, an Outerbridge class II cartilage injury of the anterior horn of the lateral meniscus (AHLM, black arrow), and lateral tibial plateau, indicating hypermobility of the $\operatorname{AHLM}(\mathrm{B})$.
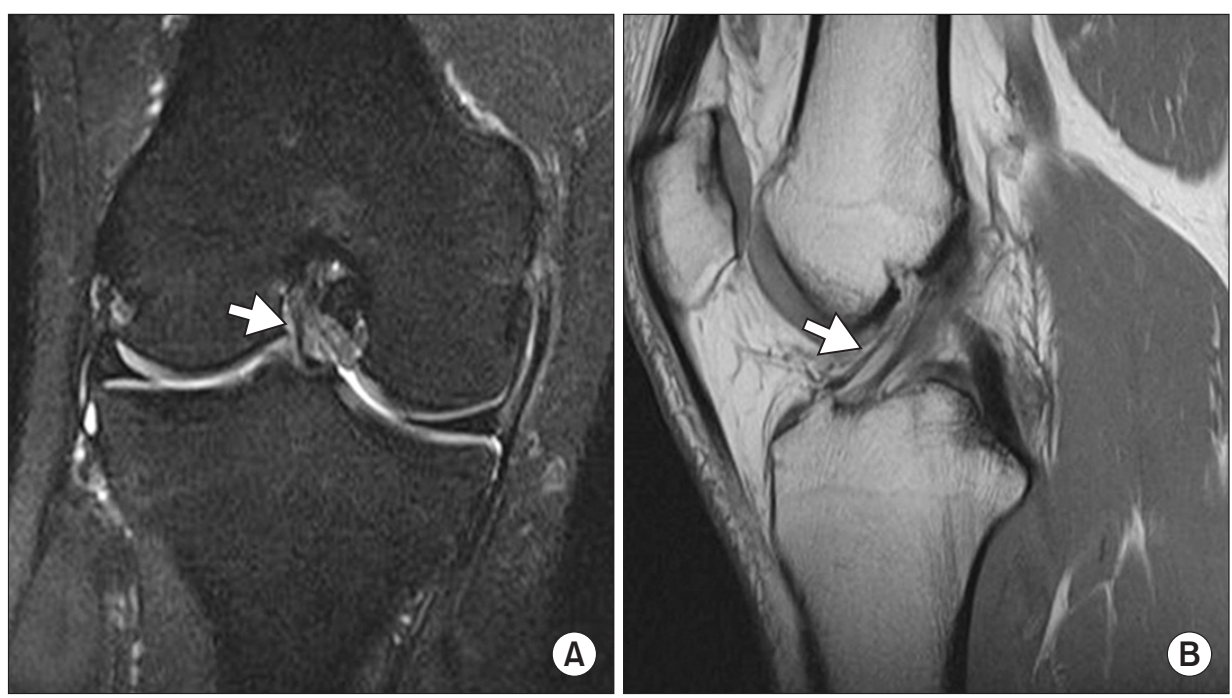

Fig. 4. Coronal T2-weighted magnetic resonance imaging (MRI) scan (A) and sagittal T1-weighted MRI scan (B) showing the anterolateral meniscofemoral ligament (white arrow).

images (Fig. 4). Because the signal intensities were similar to those of normal ligaments around the knee, we suspected it could be the anterolateral MFL that had been observed during arthroscopy. We discovered the lateral meniscus (LM) that had a normal tibial attachment of the PHLM but the AHLM lacked the tibial attachment that otherwise ran parallel to the ACL and inserted into the superior portion of the intercondylar notch anterior to the ACL. These findings were confirmed by arthroscopy. The present case also showed AHLM hypermobility, resulting in early meniscal degeneration at age 18 and an injury to the surrounding articular cartilage (Fig. 3). Since there had been no reports of this type of anomaly, we decided to administer rehabilitative treatments (e.g., strengthening exercises and activity modifications) in the outpatient clinic. Furthermore, the concomitant presence of anteromedial and anterolateral MFLs that were combined as a single structure is a new anatomical anomaly that has never been described in the literature.

The patient had been previously diagnosed with a radial tear of the LM in the contralateral knee and had undergone a partial menisectomy 10 months earlier performed by the same surgeon. No ligament abnormalities were observed in that knee on arthroscopy or MRI, indicating that it was a unilateral anomaly.

The present case report is significant in that it is the first description of an anterolateral MFL. Orthopedic surgeons should be aware of this anatomical anomaly, and future description of more cases would add to our knowledge regarding anomalous meniscus insertion.

\section{Conflict of Interest}

No potential conflict of interest relevant to this article was reported.

\section{References}

1. Kawamura S, Lotito K, Rodeo SA. Biomechanics and healing 
response of the meniscus. Oper Tech Sports Med. 2003;11: 68-76.

2. Lee DW, Ha JK, Kim JG. Medial meniscus posterior root tear: a comprehensive review. Knee Surg Relat Res. 2014;26: 125-34.

3. Jeong HJ, Lee SH, Ko CS. Meniscectomy. Knee Surg Relat Res. 2012;24:129-36.

4. Soejima T, Murakami H, Tanaka N, Nagata K. Anteromedial meniscofemoral ligament. Arthroscopy. 2003;19:90-5.

5. Gupte CM, Smith A, Jamieson N, Bull AM, Thomas RD, Amis AA. Meniscofemoral ligaments: structural and material properties. J Biomech. 2002;35:1623-9.
6. Silva A, Sampaio R. Anterior lateral meniscofemoral ligament with congenital absence of the ACL. Knee Surg Sports Traumatol Arthrosc. 2011;19:192-5.

7. Johnson DL, Swenson TM, Livesay GA, Aizawa H, Fu FH, Harner CD. Insertion-site anatomy of the human menisci: gross, arthroscopic, and topographical anatomy as a basis for meniscal transplantation. Arthroscopy. 1995;11:386-94.

8. Jung YB, Yum JK, Bae YJ, Song KS. Anomalous insertion of the medial menisci. Arthroscopy. 1998;14:505-7.

9. Ohkoshi Y, Takeuchi T, Inoue C, Hashimoto T, Shigenobu $\mathrm{K}$, Yamane S. Arthroscopic studies of variants of the anterior horn of the medical meniscus. Arthroscopy. 1997;13:725-30. 